\title{
Non-invasive assessment of pulmonary blood supply after staged repair of pulmonary atresia
}

\author{
STEFANO DEL TORSO, MICHAEL J KELLY, $¥$ VICTOR KALFF, \\ GIOVANNI STELLIN, + ROGER B B MEE, + ALEXANDER W VENABLES ${ }^{\star}$
}

From the Departments of ${ }^{\star}$ Cardiology and + Cardiac Surgery, Royal Children's Hospital; and the $\ddagger$ Nuclear Medicine Department, Alfred Hospital, Melbourne, Austratia

SUMMARY Radionuclide studies were performed to determine pulmonary blood flow in six children who had undergone surgery for pulmonary atresia, ventricular septal defect, and hypoplastic pulmonary arteries with or without major aortopulmonary collateral arteries. Lung blood flow was assessed from both particle perfusion lung scans and the pulmonary and systemic phase of a radionuclide dynamic flow study. Five patients had perfusion defects identified on the particle perfusion lung scan. In three of these, abnormal areas were perfused only during the systemic phase of the flow study, a combination of findings that indicate the presence of perfusion by collateral arteries. In one patient no systemic perfusion was noted and in one an initial particle perfusion study indicated the presence of a lung segment perfused by a collateral artery. In this last patient the particle perfusion scan after total correction showed a reduction in the size of the lung perfusion defect and no evidence of lung perfusion during the systemic phase of the flow study. The particle perfusion lung scan in the sixth patient showed pronounced asymmetry in blood flow to the lungs with no segmental perfusion defect on the particle perfusion scan and no abnormalities on the systemic flow study.

It is concluded that radionuclide lung perfusion and flow studies provide useful information on lung perfusion and merit further evaluation to define their role in the management of these patients.

There is still debate regarding the surgical management of patients with pulmonary atresia, ventricular septal defect, and hypoplastic pulmonary arteries with or without major aortopulmonary collateral arteries. ${ }^{1}$ It is, however, generally agreed that the long term results of surgery depend on achieving maximal direct segmental perfusion of the lungs and satisfactory relief of right ventricular outflow tract obstruction. ${ }^{2-4}$

After each staged palliative operation haemodynamic and angiographic investigations are usually performed. The numbers of invasive investigations should be reduced if possible as each adds morbidity and discomfort for the patient. Non-invasive radionuclide assessment of lung blood flow has the potential for this.

We report our experience with radionuclide studies

Requests for reprints to Dr Alex Venables, Department of Cardiology, Royal Children's Hospital, Flemington Road, Melbourne 3052, Australia.

Accepted for publication 22 March 1985 in determining pulmonary blood flow in six patients with this complex abnormality. Six underwent studies postoperatively and one was also investigated preoperatively.

\section{Patients and method}

Six patients were studied (age range 6-15 years). Each had pulmonary atresia, ventricular septal defect, and hypoplastic pulmonary arteries, five with and one without collateral arteries. Table 1 shows the past history and clinical data of each patient at the time of radionuclide investigation.

\section{RADIONUCLIDE DATA ACQUISITION}

After informed consent was obtained an intravenous cannula was inserted into a brachial vein. A two stage scintigraphic procedure was then performed. Firstly, a standard multiple view lung particle perfusion scan was performed using a small field of view gammacamera fitted with a general all purpose low energy 
Table 1 Patient data

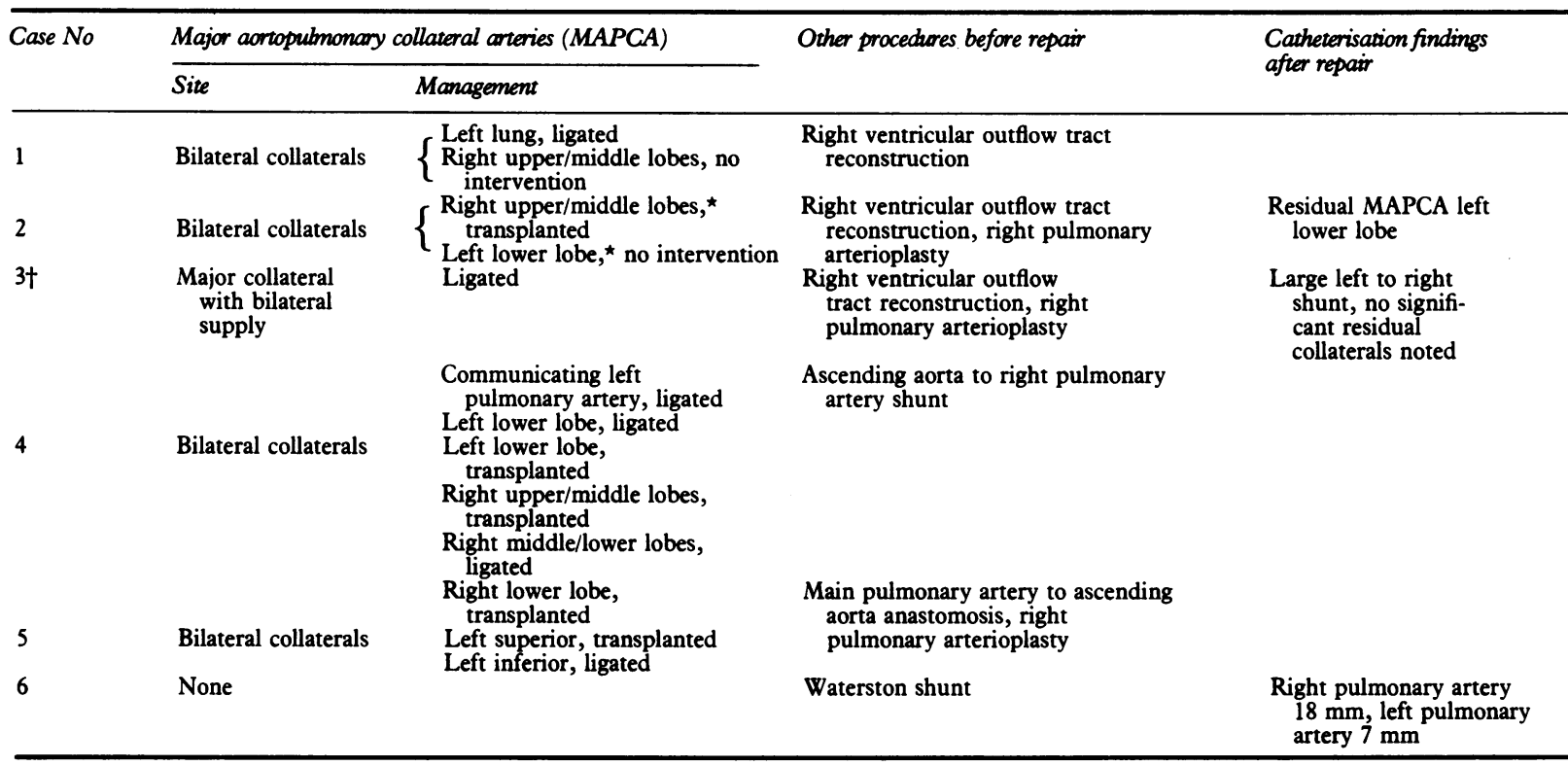

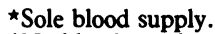

†No blood supply seen to right middle lobe preoperatively.

collimator. A dose of technetium-99m labelled macroaggregated albumin was calculated by patient weight based on an adult dose of $1 \mathrm{mCi}(37 \mathrm{MBq}$ ). With the patient lying supine, scintigraphic data were acquired on transparencies and a dedicated minicomputer (DEC 11/34 computer using Gamma-11 software). The scans initially acquired were in the anterior and left and right lateral views. If there was evidence of a right to left cardiac shunt extra scans were recorded of the head and the kidneys. This phase of the study finished with a posterior lung scan with the patient lying supine above the gammacamera.

Secondly, a dynamic radionuclide flow study was acquired immediately after the last lung particle perfusion scan and without the patient moving in relation to the gammacamera. For this study technetium-99m as pertechnetate was injected as a bolus and images were acquired on to transparencies as sequential two second views and also into the computer as a one frame per second dynamic study for 50 seconds after bolus injection. The dose of technetium- $99 \mathrm{~m}$ used was calculated according to patient weight and based on an adult dose of $20 \mathrm{mCi}(740 \mathrm{MBq}) .^{5}$

DATA ANALYSIS

A recent chest radiograph was available for each patient to help in the interpretation of the scans. The

Table 2 Radionuclide study data

\begin{tabular}{|c|c|c|c|}
\hline \multirow[t]{2}{*}{ Case No } & \multirow[t]{2}{*}{ Defect on lumg particle perfusion scan } & \multicolumn{2}{|l|}{ Dymamic heng flow scan } \\
\hline & & Pulmonary phase defect & Systemic phase visualisation \\
\hline $\begin{array}{l}1 \\
2 \\
3^{\star} \\
4 \\
5\end{array}$ & $\begin{array}{l}\text { Right upper lobe } \\
\text { Left lower lobe } \\
\text { Right middle lobe } \\
\text { Bilateral defects } \\
\text { tLeft upper lobe }\end{array}$ & $\begin{array}{l}\text { Right upper lobe } \\
\text { Left lower lobe } \\
\text { Right middle lobe } \\
\text { Normal }\end{array}$ & $\begin{array}{l}\text { Right upper lobe } \\
\text { Left lower lobe } \\
\text { Right middle lobe } \\
\text { Normal }\end{array}$ \\
\hline 5 & FSmaller defect & Normal & Normal \\
\hline 6 & Right lung: left lung flow ratio $6 \cdot 7: 1$ & Increased flow to right lung & Normal. \\
\hline
\end{tabular}

*No blood supply seen to right middle lobe preoperatively.

tLung particle perfusion study only performed after transplant of major aortopulmonary collateral artery to right lower lobe.

†Combined study performed after transplant of major aortopulmonary collateral artery to left upper lobe and total repair. 

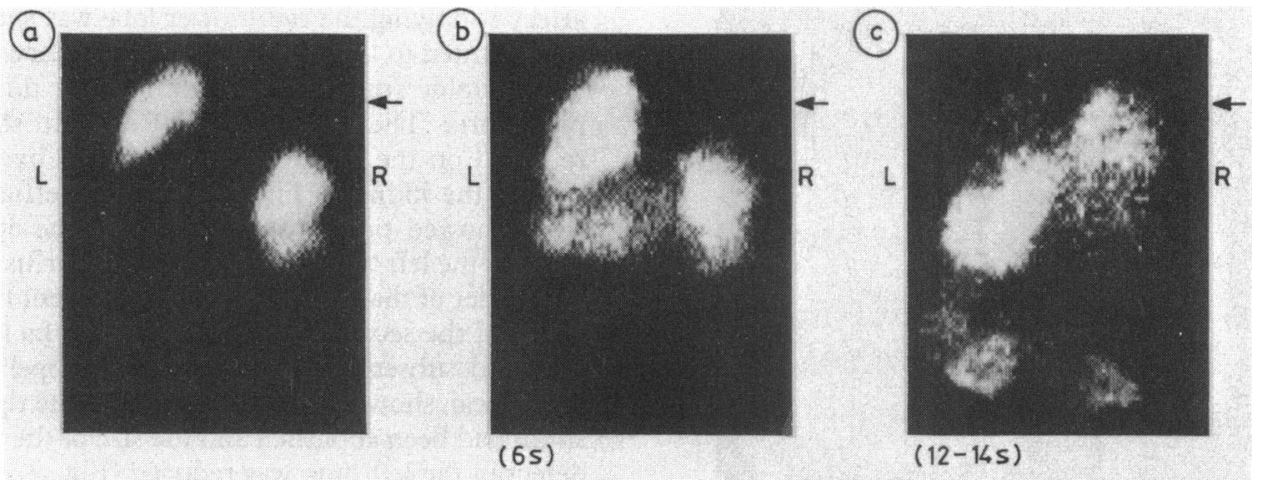

Fig. 1 Case 1: (a) particle perfusion lung scan in posterior view showing the right upper lobe perfusion defect (arrow). The perfusion defect (arrow) is also shown in (b) the lung phase of the technetivon-99 flow study, with perfusion in (c) the systemic phase of that study. $L$, left; $R$, right.

relative perfusion of each lung from the pulmonary arteries was calculated from the digital images of the particle perfusion scan as a ratio of the arithmetic mean activity of the anterior and posterior images of each lung. The particle perfusion scans were then visually examined for the presence of segmental defects representing areas of the lung that were not perfused from the pulmonary arteries.
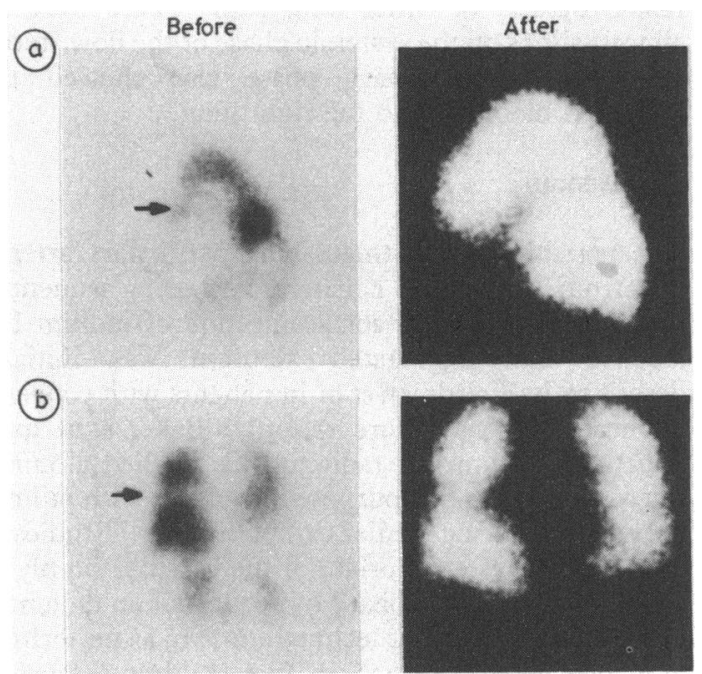

Fig. 2 Case 5: particle perfusion lung scans in (a) the left lateral and (b) posterior views before and after operation showing absent perfusion to all but the apical segment (arrow) of the left upper lobe preoperatively. The perfusion defect over the right lung was explained by a pleural effusion present on a chest radiograph. Note bilateral kidney visualisation on the preoperative posterior image $(b)$ indicating significant right to left shunting. Postoperatively the left upper lobe perfusion defect is much smaller and there is no evidence of right to left shunting.
To assess whether the defects corresponded to areas perfused by collateral arteries the first pass radionuclide flow study was reformatted as sequential one second images and then examined in a closed loop cine format in conjunction with the sequential transparencies. Perfusion of lung segments by functioning collateral arteries was inferred if the underperfused areas on the particle perfusion lung scan filled in on the radionuclide flow study only after the bolus of tracer had passed through the left ventricle and out of the aortic outflow tract in the systemic phase of the scintigraphic flow study.

\section{Results}

Table 2 summarises the results of the lung perfusion studies and ventricular function.

\section{SCINTIGRAPHIC FINDINGS}

Of the six patients, five had perfusion defects identified on the particle perfusion lung scan. In three of these, abnormal areas were perfused only during the systemic phase of the radionuclide flow study, whereas in one no systemic perfusion was noted. In the fifth patient initial scintigraphic abnormalities were reduced in a subsequent study performed after total correction. The radionuclide studies in the sixth patient showed pronounced asymmetry in blood flow to the lungs with no segmental perfusion defects on the particle perfusion scan and no abnormalities on the systemic phase of the flow study.

Case 1-This patient, who underwent repair early in our series, had had two collateral arteries, one to each lung. The right sided collateral supplied the right upper and middle lobes. No collateral artery transplant was performed, but the left sided collateral artery was ligated. At scintigraphic assessment post- 


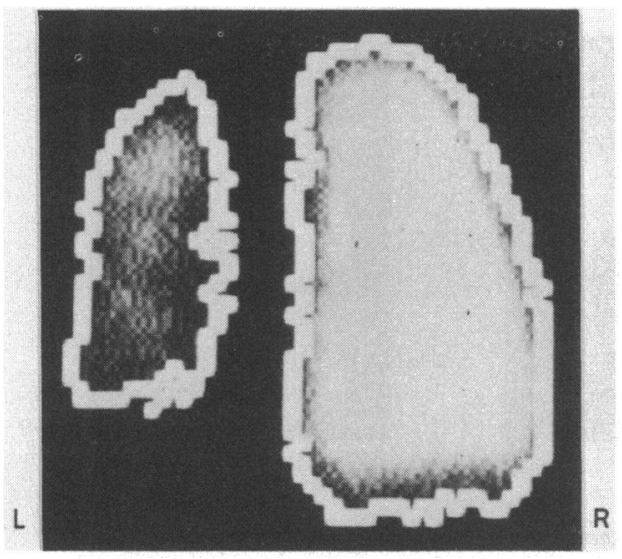

Fig. 3 Case 6: digital image in the posterior view of a postoperative particle perfusion lung scan showing relative pulmonary perfusion to right and left lungs estimated from the ratio of counts obtained within each lung field (left 48000 , right 320 000; right to left ratio 6.7:1). $L$, left; $R$, right.

operatively this patient had a perfusion defect in the right upper lobe, as seen on the posterior image of the particle perfusion scan (Fig. 1). This defect was confirmed on the pulmonary phase of the flow study (Fig. 1). Perfusion of this segment was clearly identified on the systemic phase of the flow study (Fig. 1). This finding was considered to confirm perfusion of the lung segments by the known persisting collateral artery.

Case 2-Angiographic confirmation of a collateral artery was obtained in a further patient (case 2), in whom a combination of scintigraphic findings similar to those in case 1 was observed in the left lower lobe, suggesting a blood supply of this region by a collateral artery.

Case 3-This patient had underperfused areas on the particle perfusion lung scan and on the lung phase of the flow study with systemic flow to the right middle lobe. Previous invasive studies had failed to show any blood supply to this region. This was thought to suggest an angiographically unrecognised collateral artery.

Case 4-This patient had multiple areas of underperfusion on both the particle perfusion lung scan and the pulmonary phase of the flow study. These areas did not appear to be due to perfusion by collateral arteries since they were not seen to perfuse on the systemic phase of the flow study.

Case 5-This patient had a particle perfusion lung scan before and both a particle perfusion and flow study after his final repair. At initial contrast angiography collateral arteries were identified as the sole blood supply to the right lower lobe and to most of the left upper lobe. At his first operation the collateral artery supplying the right lower lobe was successfully transplanted to the native pulmonary artery. The first scintigraphic study was performed after this surgical procedure. The expected right to left shunt was reflected on the particle perfusion scan by visualisation of the kidneys (Fig. 2). Particle perfusion lung scans showed preserved perfusion to the apical segment of the left upper lobe with underperfusion of the remainder of that lobe. He then underwent transplantation of the second collateral artery to the left upper lobe and subsequent total repair. Postoperatively, a repeat scan showed that the features of the right to left shunt had been abolished and the size of the perfusion defect in the left lung was reduced (Fig. 2). The flow study did not show any lung flow during the systemic phase to suggest residual lung perfusion by collateral arteries.

Case 6-This patient had no collateral arteries. A Waterston shunt had been performed at 3 months of age. One year after total repair a follow up cardiac catheterisation showed a pronounced difference in the size of the right and left pulmonary arteries. The lung scan showed pronounced asymmetry of blood flow to the lungs (Fig. 3) with a right to left lung perfusion ratio of 6.7 to 1.0 . The ratio of the calculated areas of the two pulmonary arteries from the cardiac catheterisation data (Table 1) was very similar to the radionuclide perfusion ratio. There were no focal perfusion defects in either lung field. There were no abnormalities on the systemic phase of the flow study, although its pulmonary phase also showed the increased blood flow to the right lung.

\section{Discussion}

The possibility of distinguishing pulmonary arterial flow from "bronchial" collateral flow using sequential intravenous and intra-aortic injection of indium-131 labelled macroaggregated albumin was initially described by Friedman et al in patients with congenital heart disease. 6 More recently, Baker et al used selective injections of radionuclide labelled albumin microspheres for this purpose in patients with pulmonary atresia during cardiac catheterisation. ${ }^{7}$ Studies to evaluate the systemic origin of the vascular supply in cases of bronchopulmonary by sequestration radionuclide angiography using technetium- $99 \mathrm{~m}$ as pertechnetate have been reported in five children. ${ }^{8}$ Particle perfusion studies have been used with intravenous injections to evaluate pulmonary blood flow in patients with tetralogy of Fallot before ${ }^{9}$ and after total repair. 910 This technique alone, however, is limited in that it provides no information on lung blood flow arising from systemic collaterals. There have been two previous reports, each describing one case, in which the combination of particle perfusion lung scans and 
radionuclide flow studies has been used to assess pulmonary blood flow more comprehensively, using intravenous injections of tracers. ${ }^{11} 12$

To our knowledge this is the first description of the use of lung particle perfusion combined with radionuclide flow studies to assess both pulmonary and systemic blood flow to the lungs in patients with pulmonary atresia, ventricular septal defect, and hypoplastic pulmonary arteries with or without collateral arteries.

The surgical management of this complex abnormality requires a precise understanding not only of the intracardiac anatomy but also of the pulmonary blood supply. ${ }^{13-15}$ Initial cardiac catheterisation is performed to obtain necessary information on the intracardiac defect, the presence or absence of central pulmonary arteries and whether they are confluent or not, their connections with the intrapulmonary arteries, the number, origin, connections, and distributions of collateral arteries, and the presence of stenosis of the pulmonary arteries or of collateral arteries.

Follow up catheterisation is usually performed before each stage of surgical repair to identify residual abnormality. Uneven growth with residual stenoses of central pulmonary arteries has also been described by Kirklin in his review of Freedom et al.$^{1}$ Right ventricular outflow tract patches, peripheral shunts, and collateral artery transplantation all have the potential of producing stenosis. ${ }^{16} 17$ There may be persistence of stenosis or occlusion of transplanted collateral arteries or residual arborisation defects due to previously unrecognised collateral arteries.

Angiographic demonstration of the central pulmonary arteries and of collateral arteries may not be fully satisfactory. ${ }^{34}$ Other techniques include computed axial tomography ${ }^{18}$ and radionuclide perfusion and dynamic flow scintigraphy. The latter appears to be a less traumatic alternative with greater sensitivity for abnormal lung blood flow. Pulmonary particle perfusion scans undoubtedly provide precise and reliable information regarding pulmonary blood flow abnormalities. The technetium- $99 \mathrm{~m}$ labelled particulate material injected is filtered out at the first downstream capillary bed encountered. Areas of decreased pulmonary arterial perfusion appear as areas of decreased radioactivity. Adequate evaluation of scintigraphic data requires correlation with a recent chest radiograph. For example, one of our patients (case 5) showed a large perfusion defect over the right lung, which was explained by a pleural effusion (Fig. 2). Right to left shunts can be detected because particles bypass the lung capillary system and radioactivity appears in the systemic circulation in brain and kidneys.

The dynamic flow study assesses both pulmonary blood flow and systemic arterial flow to the lungs. Early entry of radioactive material to the aorta as a result of right to left shunting may complicate separation of the two phases. In the absence of significant right to left shunting areas of lung perfused by collateral arteries are visualised only during the systemic arterial phase.

We have identified various combinations of patterns on lung particle perfusion scintigraphy and flow study. Firstly, areas of underperfusion seen on the particle perfusion study and pulmonary phase of the flow study, and perfused only during the systemic phase of the flow study (Fig. 1), strongly suggest the presence of lung perfusion by residual collateral arteries. This suspicion was confirmed in one of our patients at postoperative cardiac catheterisation. One other patient with a similar combination of data was known to have a collateral artery which had not been dealt with at surgery.

Secondly, areas of underperfusion seen on both the particle perfusion lung scan and the pulmonary phase of the flow study, and in which no matching perfusion is seen during the systemic phase of the flow study, indicate lung segments without appreciable blood supply of either pulmonary or systemic origin. These may be due either to ligation or occlusion of collateral arteries representing the sole supply of lung segments or to areas of lung tissue abnormality. Impaired growth of the alveoli with a reduction in their number may be caused by prolonged hypoxia and diminished blood flow. ${ }^{15}$ The small residual postoperative perfusion defect in the patient in case 5 (Fig. 2) may be of this type.

Thirdly, homogeneous pulmonary perfusion on particle perfusion scans associated with a normal systemic phase on the flow study was seen only in patients without residual functioning collateral arteries, even in the presence of asymmetrical flow due to pronounced difference in growth between the two pulmonary arteries. Digitisation of the particle perfusion scan enables relative flow to individual segments in the same lung or to each lung field to be assessed quantitatively, ${ }^{10}$ as in the patient in case 6 , in whom the pronounced asymmetry in blood flow to the lungs was due to the difference in growth between the two native pulmonary arteries. After a Waterston shunt, patterns showing either increased, ${ }^{9}$ as in the patient in case 6 (Fig. 3), or decreased ${ }^{10}$ lung particle perfusion in the lung that had received the shunt have been previously described.

Fourthly, an additional possibility, not seen in our patients, should be considered. Unrecognised or untreated collateral arteries, connected to the native pulmonary arteries, can be suspected if areas of lungs already perfused during the perfusion study appear again during the systemic phase of the flow study. 
The management of the patient with pulmonary atresia, ventricular septal defect, and hypoplastic pulmonary arteries remains a problem, in particular when collateral arteries with or without arborisation defects are present. Uniform growth of the pulmonary artery system, and unifocalisation of blood supply when collateral arteries are present, are desirable as a prelude to corrective surgery, but abnormal lung perfusion may persist even after total repair.

Repeated evaluations are necessary, and angiography is currently usually performed to assess the results of the staged surgical procedures, often after each operation. Contrast angiography can precisely define the size and growth of pulmonary arteries and is essential both in the initial preoperative evaluation and immediately before and after final repair. To repeat this investigation after every staged palliative procedure before final repair would, however, not be entirely necessary when a precise anatomical assessment of pulmonary blood vessels is not needed, if a simpler relatively non-invasive technique was readily available to assess the physiological effects of each staged procedure. Our limited comparison of the results of postoperative cardiac catheterisation with combined radionuclide blood flow studies suggests that these radionuclide techniques have some potential to identify areas of lung perfused by functioning collateral arteries and residual right to left shunts and therefore may be of value as a non-invasive follow up investigation in this clinical situation.

These scintigraphic studies may also be potentially useful in the preoperative period to direct selective angiography or to clarify further lung perfusion patterns, reducing the need for prolonged exposure to radiation from multiple contrast angiograms. A specific factor which may particularly limit the diagnostic value of this non-invasive technique before surgical intervention is the presence of major shunting from right to left (as in the initial study in the patient in case 5) or, to a lesser extent, from left to right. The pulmonary and systemic phases are not discrete under these circumstances, and inaccuracies in the quantitative prediction of the contribution of regional blood flow of the various sources would be inevitable. Because of these considerations, radionuclide methods may be of most value in the preoperative assessment of these patients when selective injection of radiolabelled particles is performed at the time of the definitive cardiac catheterisation. ${ }^{7}$

It is likely that a specific limitation of radionuclide techniques could be, for example, the difficulty in identifying perfusion by collateral arteries to the left lower lobe because of overlap with the left ventricle. Nevertheless, our data indicate that radionuclide techniques merit further evaluation to assess their possible role in the overall management of these patients, especially when collateral arteries are present.

\section{References}

1 Freedom RM, Pongiglione G, Williams WG, Trusler GA, Rowe RD. Palliative right ventricular outflow tract construction for patients with pulmonary atresia, ventricular septal defect, and hypoplastic pulmonary arteries. I Thorac Cardiovasc Sung 1983; 86: 24-36.

2 Gill CC, Moodie DS, McGoon DC. Staged surgical management of pulmonary atresia with diminutive pulmonary arteries. $\mathcal{F}$ Thorac Cardiouasc Surg 1977; 73: 436-42.

3 Alfieri O, Blackstone EH, Kirklin JW, Pacifico AD, Bargeron LM Jr. Surgical treatment of tetralogy of Fallot with pulmonary atresia. F Thorac Cardiouasc Surg 1978; 76: 321-35.

4 Haworth SG, Rees PG, Taylor JFN, Macartney FJ, De Leval M, Stark J. Pulmonary atresia with ventricular septal defect and major aortopulmonary collateral arteries. Effect of systemic pulmonary anastomosis. Br Heart f 1981; 45: 133-41.

5 Butler AM, Ritchie RH. Simplification and improvement in estimating drug dosage and fluid and dietary allowances for patients of varying sizes. $N$ Engl $F$ Med 1960; 262: 903-8.

6 Friedman WF, Braunwald E, Morrow AG. Alterations in regional pulmonary blood flow in patients with congenital heart disease studied by radioisotope scanning. Circulation 1968; 37: 74758.

7 Baker EJ, Malamitsi J, Jones ODH, Maisey MM, Tynan MJ. Use of radionuclide labelled microspheres to show the distribution of the pulmonary perfusion with multifocal pulmonary blood supply. Br Heart F 1984; 52: 72-6.

8 Gooneratne N, Conway JJ. Radionuclide angiographic diagnosis of bronchopulmonary sequestration. I Nucl Med 1976; 17: 1035-7.

9 Puyau FA, Meckstroth GR. Evaluation of pulmonary perfusion patterns in children with tetralogy of Fallot. AYR 1974; 122: 119-24.

10 Alderson PO, Boonvisut S, McKnight RC, Hartman AF Jr. Pulmonary perfusion abnormalities and ventilation-perfusion imbalance in children after total repair of tetralogy of Fallot. Circulation 1976; 53: 332-7.

11 Prosin MA, Mishkin FS. Radionuclide diagnosis of pulmonary sequestration. $f$ Nucl Med 1974; 15: 636-8.

12 Janos GG, Gelfand MJ, Schwartz DC, Kaplan S. Postoperative evaluation of the Fontan procedure by radionuclide angiography. Am Heart f 1982; 104: 785-90.

13 Thiene G, Frescura C, Borolotti U, Del Maschio A, Valente $M$. The systemic pulmonary circulation in pulmonary atresia with ventricular septal defect: concept of reciprocal development of the fourth and sixth aortic arches. Am Heart $\mathcal{F}$ 1981; 101: 339-44.

14 Haworth SG, Macartney FJ. Growth and development of pulmonary circulation in pulmonary atresia with ventricular septal defect and major aortopulmonary collateral arteries. Br Heart $\mathcal{F}$ 1980; 44: 14-24.

15 Rabinovitch M, Herrera-De Leon V, Castaneda AR, Reid L. Growth and development of the pulmonary vascular bed in patients with tetralogy of Fallot with or without pulmonary atresia. Circulation 1981; 64: 1234-49.

16 Piehler JM, Danielson GK, McGoon DC, Wallace RB, Fulton RE, Mair DD. Management of pulmonary atresia with ventricular septal defect and hypoplastic pulmonary arteries by right ventricular outflow construction. F Thorac Cardiovasc Sung 1980; 80: 552-67.

17 Lane I, Treasure T, Leijala M, Shinebourne E, Lincoln C. Diminutive pulmonary artery growth following right ventricular outflow tract enlargement. Int $\mathcal{F}$ Cardiol 1983; 3: 175-85.

18 Sondheimer HM, Oliphant M, Schneider B, Kavey RW, Blackman MS, Parker FB Jr. Computerized axial tomography of the chest for visualization of "absent" pulmonary arteries. Circulation 1982; 65: 1020-5. 